\title{
Per una linea leopardiana dell'impegno: Sulla matrice etico-politica Dell'opera di Gianni Celati degli anni Duemila
}

\section{Michele Ronchi Stefanati}

Abstract: Per lungo tempo, una certa tendenza critica è stata solita legare il concetto di impegno a quello di realismo, indicando come "letteratura dell'impegno" soltanto le scritture di rappresentazione e denuncia di una realtà sociale o politica e contrapponendola rigidamente al postmodernismo, visto come sinonimo di disimpegno. All'alba degli anni Duemila, nuovi studi hanno aperto strade diverse, cogliendo la complessità del concetto di impegno nelle sue numerose trasformazioni, individuandone linee di tendenza e momenti di rottura nel corso del Novecento, ma affermando la persistenza di motivi di critica etica e sociale anche in anni solitamente considerati di totale disimpegno e introversione. L'opera di Gianni Celati rappresenta un esempio di impegno, mai dichiarato, a cui non corrisponde nessun "ritorno al reale", anzi in aperto contrasto rispetto ad esso. Quello di Celati è un "impegno fantasticante", laddove l'intera sua opera, dagli esordi negli anni 1970 fino agli ultimi scritti, sembra partire da una considerazione critica sulla società contemporanea, sviluppando tendenze anti-autoritarie, anti-gerarchiche, anti-consumistiche e antiegemoniche espresse, però, senza alcun ricorso al realismo. Il presente studio si concentra sulle forme di impegno etico-politico nell'opera di Celati degli anni Duemila, anni che hanno dato vita, attraverso la collaborazione alla rivista Zibaldoni e altre meraviglie, a quella che si potrebbe chiamare una "linea leopardiana dell'impegno", per i numerosi riferimenti all'opera di Leopardi e al ruolo che essa ha avuto in quest'ottica.

La critica tradizionale è stata solita legare il concetto di impegno a quello di realismo, nelle sue varie accezioni, indicando come "letteratura dell'impegno" soltanto le scritture di rappresentazione e denuncia di una realtà sociale o politica e contrapponendola rigidamente al postmodernismo, visto come 
sinonimo di disimpegno. ${ }^{1}$ A ciò si aggiungeva la convinzione che l'impegno fosse irrimediabilmente scomparso sul finire degli anni Settanta, in corrispondenza simbolica con la stagione stragista e la morte di Pier Paolo Pasolini, e venisse recuperato solo dopo gli attacchi terroristici che hanno colpito gli Stati Uniti d'America l'undici settembre duemilauno, a partire da quando si sarebbe registrata, sempre secondo tale vulgata critica, una diffusa esigenza, tra gli scrittori, di un sempre più deciso 'ritorno al reale'.

All'alba degli anni Duemila, nuovi studi hanno aperto strade diverse, cogliendo la complessità del concetto di impegno nelle sue numerose trasformazioni, individuandone linee di tendenza e momenti di rottura nel corso del Novecento, ma affermando la persistenza - in letteratura e non solo - di motivi di critica etica e sociale anche in anni solitamente considerati di totale disimpegno e introversione. Jennifer Burns, nel suo capitale studio Fragments of impegno (2001), che può considerarsi il principio di questa nuova linea critica, mette in discussione le premesse teoriche su cui si era basata la critica tradizionale e prende in esame proprio il periodo 1980-2000, per dimostrare come esempi di letteratura impegnata, sotto nuove forme, continuino ad essere presenti nella narrativa italiana anche negli anni del cosiddetto 'riflusso'.

\footnotetext{
${ }^{1}$ Su questa linea i volumi e gli interventi di Romano Luperini, Lallegoria del moderno. Saggi sull'allegorismo come forma artistica del moderno e come metodo di conoscenza e, dello stesso autore, La fine del postmoderno; Alfonso Berardinelli, Casi critici. Dal postmoderno alla mutazione; Carla Benedetti, Il tradimento dei critici; Raffaele Donnarumma, "Postmoderno italiano: Qualche ipotesi" e "Ipermodernità: Ipotesi di congedo dal postmoderno." Si vedano anche il numero di Alfabeta 2 (n. 1 a. 1 del luglio-agosto 2010) dedicato all'impegno e quello di MicroMega (6, 2013) dedicato a "L'intellettuale e l'impegno."

${ }^{2} \mathrm{Si}$ vedano almeno, oltre al volume di Jennifer Burns (Fragments of impegno. Interpretations of Commitment in Contemporary Italian Narrative 1980-2000) i seguenti contributi: Pierpaolo Antonello, Florian Mussgnug, Postmodern Impegno: Ethics and Commitment in Contemporary Italian Culture; Pierpaolo Antonello, Dimenticare Pasolini. Intellettuali e impegno nell'Italia contemporanea; Elizabeth Wren Owens, Postmodern Ethics: The Re-Appropriation of Committed Writing in the Works of Antonio Tabucchi and Leonardo Sciascia 1975-2005; Eugenio Bolongaro, Mark Epstein eRita Gagliano, Creative Interventions: The Role of Intellectuals in Contemporary; Vincenzo Binetti, "Marginalità e appartenenza: la funzione dell'intellettuale tra sfera pubblica e privato nell'Italia del dopoguerra"; Lucia Re, "Pasolini vs. Calvino One More Time: The Debate on the Role of Intellectuals and Postmodernism in Italy Today". Come ha opportunamente evidenziato Burns, la crisi del concetto di impegno, così come elaborato negli anni del dopoguerra e legato alla letteratura della resistenza, andrebbe retrodatata alla fine degli anni
} 
Gianni Celati (1937-) ha rappresentato, lungo tutta la sua carriera, un esempio di impegno, mai dichiarato, non dipendente da forme di realismo e, anzi, in aperto contrasto rispetto ad esse. Forme di impegno sono dunque presenti nell'intera sua opera che, dagli esordi agli ultimi scritti, sembra sempre partire da una considerazione critica sulla società contemporanea, sviluppando tendenze anti-autoritarie, anti-gerarchiche, anti-consumistiche e anti-egemoniche che mai abbandonano lo scrittore nativo di Sondrio e sono continuamente ribadite, tanto nei libri quanto nelle interviste e negli altri interventi pubblici. ${ }^{3}$ L'opera di Celati può vedersi sempre come una "testarda protesta sovversiva" (Celati, Finzioni 202), ${ }^{4}$ tanto negli anni di Comiche e delle figure marginali ${ }^{5}$ che affollano i primi libri, quanto in quella "poetica del reincanto" (nell'epoca del disincanto) (Rizzante 69), volta a salvaguardare l'istinto narrativo sempre in netta contrapposizione alle abitudini industriali dell'editoria, che domina da Narratori in poi; ${ }^{6}$ una "narrativa delle riserve" che ha avuto larga influenza sulla letteratura contemporanea, fungendo da modello, anche etico, per le generazioni successive a Celati. ${ }^{7}$ Non

Sessanta(13-35); in queste pagine la studiosasi sottolinea come siano gli stessi protagonisti di quel tipo di impegno a dichiararne la fine e a cercare forme nuove di critica sociale.

${ }^{3}$ Sull'impegno in Celati si vedano: Pia Schwarz Lausten, "Impegno e immaginazione nell'opera di Gianni Celati"; Marina Spunta, "Verso la foce con Gianni Celati: tra 'antropologia di popolazioni invisibili' e 'estetica del reincanto"” e Giulio Iacoli, "Lo sguardo in macchina. Note sull'immaginario cinematografico di Gianni Celati.”

${ }^{4}$ Celati riprende qui la definizione di Walter Benjamin a proposito del collezionista Finzioni.

${ }^{5}$ Sul concetto di margine, in relazione a quello di archeologia, così centrale in questi anni sia per Calvino e Celati che per gli altri redattori della rivista (e più vicino alla riflessione di Walter Benjamin in Angelus Novus che alle formulazioni di Michel Foucault) si vedano Gianni Celati, "Il bazar archeologico" (Finzioni 195-227); Italo Calvino, "Lo sguardo dell'archeologo" e le lettere tra Celati, Calvino e Carlo Ginzburg contenute in Riga 14 (Barenghi e Belpoliti 162-167).

${ }^{6}$ L'opera di Celati mostra una straordinaria coerenza interna tra periodi diversi e lontani nel tempo che ci porta ad escludere la divisione rigida del suo lavoro in due fasi distinte e quasi contrapposte, prima e dopo Narratori delle pianure (1985). È vero però che l'incontro con Luigi Ghirri ha sviluppato elementi (sia pure già presenti) in direzioni precedentemente inesplorate e la stessa scrittura di Celati lo dimostra. Condividiamo dunque l'impostazione esposta da Rebecca West nella sua monografia su Celati.

${ }^{7}$ Narratori delle riserve è il titolo dell'antologia uscita a puntate sul Manifesto tra il 30 ottobre 1988 e il 25 luglio 1990 e poi pubblicata da Feltrinelli (1992). Il titolo è spiegato così da Severino Cesari, nel giorno dell'esordio della rubrica sul Manifesto: "Riserve nel senso di far 
stupirà, dunque, se uno dei principali fautori di una "letteratura che dica la verità" (Di Stefano), Goffredo Fofi, sia stato tra i principali oppositori di Celati e del gruppo di scrittori che ne condividono l'idea di letteratura. ${ }^{8}$

Il presente studio si concentra sulle forme di impegno etico-politico o etico-estetico (Spunta 167) nell'opera di Celati degli anni Duemila, anni che hanno dato vita, attraverso la collaborazione alla rivista Zibaldoni e altre meraviglie, a quella che si potrebbe chiamare una "linea leopardiana dell'impegno." Tratteremo in particolare il ciclo di Vecchiatto, per il quale dovremo fare un passo indietro, partendo da un testo del 1996, Recita dell'attore Vecchiatto nel teatro di Rio Saliceto, e arrivando a Sonetti del Badalucco nell'Italia odierna (2010), e faremo rapide ma decisive incursioni negli articoli apparsi in questi anni, a firma di Celati e di altri, sulla rivista Zibaldoni.

Nel suo studio, Burns indica come momento di svolta tra l'engagement del dopoguerra - organico, nato dal momento collettivo della resistenza partigiana e in continua relazione con il partito, il PCI - e le nuove forme di impegno frammentario che sorgono a partire dalla fine degli anni Sessanta la riflessione nata attorno al progetto di rivista Alì Babà nel periodo 1968-1972.; ${ }^{10}$ le implicazioni di tale esperienza sul concetto di impegno sono state studiate da Monica Francioso in un saggio apparso su Italian Studies nel 2009. ${ }^{11}$ Gianni Celati è stato,

riserva, di non consumare via tutto per darlo in pasto all'attualità." (Severino Cesari, 'L'artigiano delle parole', il Manifesto, 8 marzo 1989, p. 15). Tra i tanti scrittori che hanno riconosciuto il loro debito nei confronti dell'opera di Celati si vedano: Enrico Palandri, Pier Vittorio Tondelli, Grazia Verasani, Silvia Ballestra, Ermanno Cavazzoni, Daniele Benati, Ugo Cornia, Jean Talon, Paolo Nori, Gianluca Morozzi.

${ }^{8}$ Fofi aveva attaccato la rivista Il Semplice fondata da Celati, Ermanno Cavazzoni e altri: "In un mondo che se ne cade (e se ne cade! Se ne cade!...) ci sono letterati [...] che si contentano di tirarsi da lato (ma mantenendo tantissime complicità con il mondo via università, media, editoria...) e giocherellare con un loro modesto e scipito fantasticare" (citato in Di Stefano). Fofi ha scoperto e lanciato, fra gli altri, Roberto Saviano, probabilmente il maggiore esempio di 'ritorno al reale' nella letteratura italiana degli anni Duemila.

${ }^{9}$ Tale proposta di definizione riprende il titolo di un articolo di Celati apparso su Zibaldoni: "La linea leopardiana della prosa."

${ }^{10}$ I materiali relativi al progetto sono stati raccolti da Mario Belpoliti e Mario Barenghi in Riga 14. “Alì Babà.” Progetto di una rivista 1968-1972.

${ }^{11}$ Mi riferisco a Monica Francioso, "Impegno and Alì Babà: Celati, Calvino and the Debate on Literature in the 1970s." Si vedano anche Monica Jansen e Claudia Nocentini, "Alì Babà and 
insieme a Italo Calvino, il principale animatore del progetto per Alì Babà, a cui hanno collaborato anche Carlo Ginzburg, Guido Neri e Enzo Melandri. Come ha opportunamente notato Francioso, il principale interlocutore a cui i redattori di Alì Babà si rivolgono sembra essere il movimento studentesco. In uno dei primi protocolli, scritto nel dicembre 1968, leggiamo:

Si constata senza recriminazioni il fatto che i giovani e gli studenti che lottano non si interessano di letteratura (almeno in Italia), né trovano in alcun modo nella letteratura stimoli e parole politiche; ciò permette di capire che il denominare comune delle produzioni letterarie è troppo ristretto per soddisfare esigenze più vaste che la prassi promuove. $\mathrm{E}$ allora i letterati si mettono a parlare anche di politica (come avviene su Quindici) generalmente improvvisando o ricadendo negli slogan dei linguaggi di partito. (Barenghi e Belpoliti 57)

Al di là delle contingenze storiche e dell'insoddisfazione per la produzione letteraria coeva (segnatamente, quella del Gruppo '63), la spinta che muove i redattori di Alì Babà è tutta interna alla letteratura, dove trovare "stimoli e parole politiche." (Barenghi e Belpoliti 57). Come fare questo? Come - era questo l'intento dei redattori - recuperare il senso di "una letteratura che dica qualcosa di più, oltre al solito tran-tran dei premi letterari"? (Celati, "Il progetto", 313)..$^{12}$ Riportandola alle origini - è la loro risposta — come istinto narrativo e fabulazione, che ha a che fare con una tradizione letteraria (scritta e orale) e con le sue fonti archetipiche. In tal senso, la letteratura dovrà uscire dal suo stretto recinto e tornare a confrontarsi col mondo: "Diviene necessaria la ridefinizione della letteratura come luogo di significati e di forme che non valgono solo per la letteratura, con altri termini: come luogo dei fondamenti mitici dell'operare umano" (Barenghi e Belpoliti 58).

Una "poetica del discorso umano" che "diviene automaticamente politica" (Barenghi e Belpoliti 60) perché recupera il mito come strumento di comprensione di ogni ambito del vivere e dunque valido anche (ma non solo) per quello politico. È un tentativo di ridare centralità alla letteratura, allora più che mai schiacciata sul

Beyond: Celati and Calvino in the Search for 'Something More" e Anna Botta, "The Alì Babà project (1968-1972): Monumental History and the Silent Resistance of the Ordinary."

${ }^{12}$ Cfr. anche Italo Calvino, "Per una letteratura che chieda di più (Vittorini e il '68)," (Calvino 238-241). 
discorso politico e quindi incapace di confrontarsi con esso. Quello che manca, per i redattori di $A l i ̀ ~ B a b a ̀$, è la "dimensione immaginaria dell'emergenza politica," cioè la "strumentazione tecnica e concettuale che permetta d'investire immaginativamente i fatti che accadono sotto i nostri occhi" (Barenghi e Belopoliti 57). Celati e gli altri redattori della rivista pensano dunque alla letteratura come forma di comprensione del mondo attraverso le categorie immaginative del mito e dell'archetipo, non come appiattimento cronachistico sul reale.

Questi stessi concetti riecheggiano, a quasi quarant'anni di distanza da Alì Babà, in un intervento, apparso sul quotidiano l'Unità il 31 gennaio 2004, a firma dei redattori della rivista Zibaldoni e altre meraviglie, Enrico De Vivo e Gianluca Virgilio, dal titolo "La letteratura condivisa: il piacere di fare politica," che potremmo dire fondatore di quella che abbiamo chiamato "linea leopardiana dell'impegno":

A dispetto di decenni di critica che opponeva ingenuamente l'impegno del realismo al disimpegno del fantastico, noi abbiamo intravisto nella scrittura leopardiana dello Zibaldone di pensieri un'apertura impensata sul mondo - apertura che non implica mai una piatta rappresentazione del reale, ma coglie e suggerisce le trasformazioni attraverso visioni che danno sollievo e indicano una strada da seguire. [...] Nel solco dell'esperienza leopardiana, noi riteniamo che la letteratura oggi abbia bisogno di uno slancio visionario che la porti al di là dei suoi stessi discorsi, che sempre più spesso sono ormai solo discorsi tecnicistici e autoreferenziali. (De Vivo e Virgilio)

Si tratta, dunque, per i redattori di Zibaldoni di "far uscire la letteratura dal suo ghetto," per riprendere le parole usate da Celati a proposito dell'altra rivista, Alì Babà, nella lettera a Mario Barenghi e Marco Belpoliti scritta nel 1997 (Celati, "Il progetto" 313), dove si legge anche:

Ma siccome quel "qualcosa di più" che si andava cercando fuori dal ghetto riguardava la sua funzione (la funzione della letteratura), allora si trattava di chiedersi a che cosa servono i libri, i romanzi, le poesie, e scavare il terreno sotto i fondamenti che sono dati per scontati, e rimuginare su tutto, e infine viaggiare tra le astrazioni più vertiginose. (Celati, "Il progetto" 313) 
Se lo sforzo a recuperare il "ruolo sociale della immaginazione" (Barenghi 17) ${ }^{13}$ accomuna le due esperienze, sia pure cronologicamente così lontane, un elemento di novità rispetto all'esperienza di $A l i ̀$ Babà è costituito ora dal richiamo a Giacomo Leopardi. Perché, dunque, Leopardi?

Il nesso tra lo Zibaldone di pensieri e, più in generale, tra lo sfondo teorico dell'opera leopardiana e l'impegno politico sembra essere soprattutto, nell'idea dei redattori di Zibaldoni, nell'aperto contrasto alle tendenze alla mercificazione proprie della società contemporanea, che Leopardi criticava al suo nascere:

Leopardi, anticipando Baudelaire di qualche decennio, possiede una precisa consapevolezza politica della solitudine in cui si avvia a operare l'artista, che nell'epoca della Restaurazione comincia a esser condannato al "mercato," ossia alla servitù nei confronti di qualcosa di troppo grande ed estraneo che lo sovrasta e controlla, lo aliena e avvilisce. (Celati, "La linea leopardiana")

A fronte di questa solitudine, Zibaldoni propone un nuovo senso di comunità, in cui la letteratura e l'intellettuale giocano un ruolo centrale, pur all'interno di una rivista-zibaldone "divagante e collettiva," che si ispira alla scrittura leopardiana dei pensieri, così come descritta da Celati in un intervento uscito proprio su Zibaldoni nel 2003 e intitolato "La linea leopardiana della prosa" ("linea sempre erratica e frammentaria, mobile e sospesa"). Zibaldoni propone un impegno disorganico e asistematico, i cui obiettivi politici sono dichiarati esplicitamente nella lettera-manifesto dei redattori:

[È] un bene che gli scrittori e gli intellettuali si incontrino e discutano, perché così facendo riscoprono la nobilissima arte della Politica. Oggi non basta più il "piacere" solitario (dei libri, della lettura, etc) e l'"intelligenza" individualistica delle cose che riguardano la letteratura. Desideriamo agire per preservare quel "piacere" e quella "intelligenza" dalla distruzione prodotta dal "mercato" e dalla "bassa politica," che foraggiano una pratica letteraria lesiva della dignità dei

\footnotetext{
${ }^{13}$ Uso le parole di Mario Barenghi a proposito di Alì Babà. Cfr. Mario Barenghi, "Congetture su un dissenso."
} 
lettori e degli scrittori, e ricercare un nuovo senso comunitario della letteratura: questo significa per noi fare Politica. (De Vivo e Virgilio)

La "linea leopardiana della prosa" è la formula che Celati usa per descrivere il modus scribendi di Leopardi nello Zibaldone di pensieri, ma essa sembra indicare anche una linea scrittoria (delle riserve) che lascia ancora spazio all'immaginazione, contro le narrazioni appiattite sul reale, dominanti nel mercato editoriale degli anni Duemila:

Quello che conta non sono le mete a cui arriviamo, ma il continuo transito attraverso gli stati di affezione che sorgono, come una mobilità eccitatoria che è l'anima di questa scrittura, e di ogni modo di scrivere non ancora catturato dalle "rappresentazioni del reale."

Le frasi prescritte, il pensiero confezionato, hanno perso il ricordo di questa mobilità eccitatoria delle parole, che in Leopardi viene in primo piano. (De Vivo e Virgilio)

Sono concetti particolarmente cari a Celati e su cui lo scrittore torna più volte negli anni al centro della nostra analisi. In una conversazione con Luca Sebastiani del giugno 2009, Celati parla della rivoluzione operata da Margaret Thatcher:

Quando ho cominciato a scrivere, quasi tutti gli amici che avevo erano di sinistra e davano per scontato una rivoluzione imminente o futura. Ma l'unica rivoluzione che poi ho visto è stata quella della signora Thatcher, primo ministro inglese dal 1979. Tutto era pronto perché succedesse quello che doveva succedere: l'avvento al potere del capitalismo finanziario che schiaccia tutte le forme di produzione (industriale o artigianale), e poi della new market economy con cui il profitto diventa l'unico ideale sulla terra. (Celati, Conversazioni 142)

All'interno di questa nuova ideologia diventata l'unica ideologia, Celati colloca lo sviluppo della letteratura, dall'esperienza di Alì Babà ai cannibali. Le tendenze sdoganate dalla rivoluzione della Thatcher ("le mete oscure del potere finanziario, la considerazione del lavoro come fatto secondario, l'abolizione d'ogni veduta comunitaria, la guerra individuale per passare davanti agli altri” Celati, Conversazioni 142) hanno travolto, secondo Celati, anche la letteratura: 
E posso dire anche quando sono arrivate in Italia, e come hanno cominciato a condizionare tutto ciò che si chiamava letteratura. Quando sono stato cooptato dalla casa editrice Einaudi come traduttore e futuro autore, io non pensavo neanche per un momento alla faccenda dei soldi e del successo. E ricordandomi tutto il tempo consacrato al progetto d'una rivista che io e Italo Calvino dovevamo fare con altri, ora mi sembra qualcosa di irreale [...]. Tutto questo lavoro aveva un carattere gratuito, come un incontro senza idee di profitto [...].

La prima avvisaglia d'una mutazione è stata l'avvento della letteratura giovanile $[\ldots]$. E mentre la signora Thatcher apriva la nuova era, da noi iniziava la caccia all'autore giovane. Qui sono scattati tutti i meccanismi del futuro: il trattamento dello scrivere come merce, il nome dell'autore come feticcio, le etichette stantie con cui parlarne, la competizione tra case editrici. (Conversazioni $142-143)^{14}$

In aperto contrasto rispetto a quelli che altrove chiama i "fottuti romanzi industriali," ("Letteratura", 28) ${ }^{15}$ Celati prosegue, negli anni Duemila, il suo artigianato narrativo, tanto in letteratura quanto nel cinema ${ }^{16}$ e nel teatro. ${ }^{17}$

\footnotetext{
${ }^{14}$ La radicalità del pensiero di Celati su questo tema si misura anche più avanti nel testo: "Tutto ciò va insieme a una baldoria di consumi, dove il consumo di libri non si distingueva da quello delle saponette. E sarebbe bello poter pensare che un giorno ci sarà un processo alla corte dell'Aja, dove le anime dei professionisti dell'editoria saranno imputate di genocidio letterario, e massacro dell'antica tradizione dell'arte verbale delle nostre terre" (Conversazioni 144).

${ }^{15}$ Parlando della peculiarità di Verso la foce rispetto al resto della sua produzione Celati dice: "Da me, niente storie, niente trame, basta! La gente vuole romanzi, e il mio era semmai una reazione all'oscurantismo dei fottuti romanzi industriali, che hanno gli stessi caratteri cancerogeni dei prodotti chimici con assuefazione istantanea" ("Letteratura" 28).

${ }^{16}$ Celati è regista di quattro documentari usciti tra il 1991 e il 2010: Strada provinciale delle anime (1991), Il mondo di Luigi Ghirri (1999), Visioni di case che crollano - Case sparse (2003) e Diol Kadd. Vita, diari e riprese in un villaggio del Senegal (2010).

${ }^{17}$ Cfr. Celati, Bollettino del diluvio universale - Pantomima in due atti. Anche Recita dell'attore Vecchiatto è stato portato in scena da Mario Scaccia e, successivamente, dallo stesso Celati e da Nunzia Palmieri, nell'ambito delle celebrazioni dedicate allo scrittore dalla città di Bologna nel 2013 (il titolo della manifestazione, durata diversi mesi, era "La dispersione delle parole. Omaggio a Gianni Celati”).
} 
Il ciclo di Vecchiatto, composto da Recita dell'attore Vecchiatto nel teatro di Rio Saliceto e da Sonetti del Badalucco nell'Italia odierna,${ }^{18}$ costituisce il ritorno di Celati alla narrativa, a sette anni da Verso la foce. ${ }^{19}$ Sia Recita che Sonetti sono forme miste, la prima mette insieme un testo teatrale, ${ }^{20}$ quarantadue sonetti e una nota biografica fantastica inseriti in coda al libro, il secondo è un prosimetro che alterna i sonetti attribuiti all'attore internazionale Attilio Vecchiatto al racconto della sua vita e alla testimonianze su di essa di Enrico De Vivo. Di Vecchiatto, dunque, si immagina l'intera carriera attoriale, dalla fuga dall'Italia nel 1932 seguita a una rissa con una squadraccia fascista, alla rocambolesca fuga a bordo di un mercantile inglese in partenza per l'Argentina, fino alla nuova vita da attore vagante, prima a Buenos Aires, poi in tutto il Sudamerica, negli Stati Uniti, in Canada e di nuovo in Europa, a Parigi, prima da solo e poi in coppia con la moglie Carlotta Benedetti, sposata nel $1942 .{ }^{21}$ Recita è la trascrizione dell'ultimo spettacolo di Attilio Vecchiatto e di Carlotta, ormai vecchi, tornati in Italia cinquant'anni dopo

${ }^{18}$ Come ha notato Stefano Bartezzaghi, questi titoli insolitamente lunghi nascondono, sotto forma di anagramma, il cognome Celati, che ricorre quattro volte in Recita e una nel nome del suo protagonista, Attilio Vecchiatto. Aggiungiamo qui che la stessa cosa accade in Sonetti, con il nome Celati che si ritrova due volte nel titolo.

${ }^{19}$ Prima di Recita escono infatti: Parlamenti buffi (1989), riscrittura di tre libri di Celati degli anni Settanta (Le avventure di Guizzardi, La banda dei sospiri e Lunario del paradiso) e L'Orlando innamorato raccontato in prosa (1994), a sua volta riscrittura del poema boiardesco. Di questo nuovo silenzio narrativo (il primo aveva separato Lunario, del 1978, da Narratori delle pianure, 1985, di altri sette anni) si possono vedere le motivazioni nell'intervista a Celati di Belpoliti e Cortellessa: "Verso la foce è stato il terminale di tutti i miei lavori, e ho perduto l'orientamento [...]. Ricordo un editore che mi diceva con aria da connaisseur: 'Quel libro non doveva scriverlo.' Voleva dire che ero andato fuori dal seminato, e non c'entravo più niente con la letteratura" (Celati, "Letteratura" 30). Mentre in Recita si legge: "CARLOTTA: Signora, mio marito ha sempre scritto molto, non ci riesce più..." VECCHIATTO: "Tutto adesso suona falso e meschino ai miei orecchi, tutto senza gloria e senza onore..." (Celati, Recita 29). E ancora: "Ma cosa serve scrivere, impantanati nelle parole dei giornali che avvelenano tutti i pozzi, seccano ogni polla sorgiva, ammorbano l'acqua d'ogni fonte spirituale" (Celati, Recita 34).

${ }^{20}$ Si tratta di una ricostruzione dell'ultima recita di Vecchiatto, inattendibile perché basata su una registrazione fallace. Cfr. la Nota che precede il testo (Celati, Recita 9).

${ }^{21}$ Tali peregrinazioni ricordano il percorso di altre celebri coppie di teatro: Judith Malina e Julian Beck, Dario Fo (citato scherzosamente in Celati, Sonetti 41; 70) e Franca Rame. 
averla lasciata. ${ }^{22}$ Della fama acquisita nel mondo, però, in Italia non c'è traccia: in patria nessuno conosce i due attori. Al loro spettacolo assistono in pochi, incapaci all'ascolto, tutti di passaggio come da un bar all'altro o da un canale televisivo all'altro. ${ }^{23}$ La sola spettatrice attenta è una signora anziana con la borsa della spesa; ${ }^{24}$ sul finale, però, anche la signora si addormenta, e il sonno cala persino su Carlotta. Vecchiatto è ormai "fuori moda," il suo testo, di cui cadono continuamente a terra le pagine, "non regge" (Celati, Recita 23) Nel suo sfogo iniziale, prima di cominciare la recita vera e propria, continuamente interrotta, Vecchiatto prende di mira l'Italia: "Questo è un paese di abbruttiti, diciamolo pure![...] Di pazzi che si odiano tra loro. [...]Suburra del vestiario di moda! Vetrina della più zotica esibizione di ricchezza! Questo paese, nazione chiamata Italia, patria? Macché patria d'Egitto! Stige di putridi governanti! Regno della notte dell'anima!" (Recita 33).

L'Italia è descritta come "giardino invaso da male piante" (Celati, Recita 59), dove dominano il conformismo ("Il giovane maschio stordito segue come tutti la corrente $[\ldots]$. Basta che tutto sia previsto secondi i patti, le abitudini o le frivole illusioni della gente ricca" Celati, Recita 59), l'adulazione a fini di carriera ("una sera dopo lavoro si trattiene nell'ufficio per leccare un po' i piedi [...] al suo capufficio o direttore dell'azienda...e questo gli fa delle promesse" Celati, Recita

${ }^{22}$ Delle motivazioni del ritorno in Italia ne scrive De Vivo in Sonetti: "Una volta, con tono molto serio, mi spiegò perché era tornato in Italia — era tornato perché da noi, soprattutto nel Sud, si vedevano ancora per le strade dei cani randagi ('Ma ahimè, minacciati più che mai dal Badalucco,' sospirava, 'perché i cani randagi non rendono soldi alle banche...')." (Celati, Sonetti 22). Cfr. anche il distico finale del sonetto 2: "Questo è l'ordine sociale, cosiddetto: / io sto coi can randagi e i senza tetto” (Celati, Sonetti 14).

${ }^{23}$ Così Attilio maltratta il suo secondo spettatore: "No no, dica lei, perché arriva in ritardo? Crede di venir a guardare la televisione in un bar?” Il pubblico, per Vecchiatto, è freddo, apatico, perché "ottenebrato dal comfort" (Celati, Recita 47): gli spettatori "vengono a teatro annoiati di benessere" (Celati, Recita 40). Cfr. su questo Sonetti: "O spettatore frigido e annoiato / che siedi in poltrona per godere, / satollo del denaro guadagnato, / per te misura delle cose vere” (103).

${ }^{24}$ Su questa immagine della signora con la borsa della spesa Celati torna più volte, non solo nel ciclo di Vecchiatto. Particolarmente rivelatore è il seguente passaggio dell'intervista a Celati fatta da Sarah Hill: "Quando è scoppiata la guerra in Iraq ero qui a Chicago, e per una settimana ho passato le giornate guardando la televisione [...] non ho sentito una sola frase che non fosse propaganda patriottica. A un certo punto non ne potevo più e dovevo scappar fuori [...]. Poi fuori c'era il mondo, le case, le vecchie signore con l'artrite nel supermercato, i quartieri disastrati del ghetto. Ma tutto questo era come se non esistesse, spazzato via dalla fiction della guerra" (Celati, "Documentari imprevedibili" 55). Concetti simili si trovano in Jean Baudrillard. 
60) e l'accettazione passiva di qualunque regime politico (non c'è reazione "anche se il più turpe governo guida ogni nostro gesto nelle reti della pubblica impostura" Celati, Recita 61).

L'invettiva di Vecchiatto non risparmia nessun aspetto della vita italiana, dalla famiglia ("un eterno mugolarsi alle spalle [...]. Non se ne può più di questa vita familiare da topi, signora!" Celati, Recita 54), all'imposizione di un "ottimismo aziendale obbligatorio" (Celati, Recita) fino ai giornali ("i più abominevoli della terra" Celati, Recita 23). Su questi ultimi Vecchiatto insiste moltissimo, in più punti della Recita, citando ripetutamente uno dei più venduti, il Corriere della sera, e ipotizzando di incriminarli tutti perché "responsabili d'uno stato d'abbruttimento generale con le loro parole nauseabonde" (Celati, Recita 55). I giornali, secondo Vecchiatto, "assaltano i pensieri dei cittadini" e tramite essi "tutto diventa torbido, pubblico, senza più pensieri nostri, solo frasari dell'ordine pubblico" (Celati, Recita 31). ${ }^{25}$ Nulla di quello che i giornali scrivono va preso per vero: "Signora, lei crede ai fatti riportati sui giornali? Crede alle statistiche? Crede all'informazione? Sono aria fritta, signora, neanche una parola vera! Sono fantasime, bubbole della vergogna umana, che fanno marcire lo spirito, ci spingono sempre più nel ghiaccio dei gironi infernali!" (Celati, Recita 35).

La critica prosegue, se possibile più aspra ancora, nei Sonetti, dove i componimenti diventano cinquantuno. Al suo ritorno in patria, Vecchiatto trova un paese "inospite, triviale, deturpato / in mano a furbi senza alcun pudore" (Celati, Sonetti 13). L'attore prende ora di mira il mondo politico con la sua propaganda pubblicitaria e il suo marketing elettorale (sonetti 10, 14 e 15), l'effetto obnubilante della televisione (sonetti 11,12, 40), il consumismo e la dittatura del nuovo e del vincente, dove il vecchio e il perdente è rimosso (sonetti 9, 34, 41, 50), tocca il tema del potere criminale (sonetto 2 ), attacca il mondo universitario e dei critici letterari (sonetti 26, 39), l'avidità diffusa (sonetto 31), l'ottimismo obbligatorio (sonetto 33), l'apatia tecnologica (sonetto 35). Ne viene fuori un ritratto dove è ampiamente riconoscibile l'Italia degli anni Duemila, quella dominata dal berlusconismo, ${ }^{26}$ ma i cui caratteri sono universali:

\footnotetext{
${ }^{25}$ Di questi, Celati cita "pensionato" (Celati, Recita 18; 68) e "successo" (Celati, Recita 48).

${ }^{26}$ Sul berlusconismo come periodo storico e come tendenza culturale si veda Paul Ginsborg, Berlusconismo. Analisi di un sistema di potere.
} 
10.

Siate liberi - dice Badalucco -

io do la libertà, ${ }^{27}$ voi mi date i voti;

la libertà è il profitto per chi ha doti,

e senza doti niente, questo è il succo,

$[\ldots]$

se sei furbo coi quattrini tu ti quoti

$[\ldots]$

Io ho l'arte degli affari e del pilucco,

e per farvi piluccar profitti ignoti, vi do la libertà, voi mi date i voti, che i fessi ci resteranno di stucco. (Celati, Sonetti 27)

I sonetti 14 ("Sulla fabbricazione degli uomini politici attuali") e 15 ("Parlare senza non credere a niente") mostrano le modalità di reclutamento e formazione della classe dirigente, tutte fondate su modi servili e menzogneri, gli stessi con cui ci si garantisce un successo elettorale dietro al quale, dopo il voto, resta soltanto il vuoto:

14.

Questi automi stanno dritti solamente

se ben pompati e ben pubblicizzati;

[...]

s'appoggiano ai palloni più gonfiati

per far carriera da automa dirigente. (Celati, Sonetti)

15.

Questi automi, messi a governar la gente

(pencolando qua e là come ho già detto)

Tutto trasforman nell'assoluto niente:

e ora spiego come ciò va a effetto.

\footnotetext{
27 Polo delle Libertà (1994-1996), Casa delle libertà (2000-2008) e Popolo della libertà (2008-2013) sono state le alleanze e il partito che hanno sostenuto l'ascesa di Silvio Berlusconi dal 1994 in poi (ma si veda anche il precedente del nazionalista Partito della Libertà Austriaco - FPÖ — di cui fu leader, dal 1986 al 2000, Jörg Haider). Una rappresentazione satirica di Berlusconi è riconoscibile in vari punti dei Sonetti. Cfr., tra i tanti esempi: "Fai (come Badalucco) del tuo riso; / uno stampo cosmetico lucente" (Celati, Sonetti 26).
} 
Un sì o un no per loro è equivalente, e conta solo nell'attimo in cui è detto, per far carriera e mostrarsi ubbidiente al branco in cui l'automa verrà eletto. (Celati, Sonetti 37)

La perenne rincorsa di un guadagno facile e cospicuo è la principale preoccupazione in questa Italia ("di morire in pace ormai nessuno spera / perché fino all'ultimo ha pretese / che il denaro lo salvi dalla fiera / morsa del nulla che sempre lo attese" Celati, Sonetti 66), dove la "belva finanziaria e menzognera / elegge a dio il denaro d'un paese" (Celati, Sonetti 66) e spinge anche a forme di arricchimento disonesto, tra omertà, omicidi e cementificazione:

2.

Ecco la piana di Aversa, che ora consta

Di scheletri in cemento e case orrende, create per il lucro di una cosca che fa, disfa, massacra e tutto svende.

Peggior danno non c’è che si conosca: inferno, galera, ricatto che tende a chiudervi la bocca, nella losca congrega di assassini che rivende l'anima vostra, urlando: "Zitto e mosca!."

Così arricchirete in orride faccende, homo homini lupus, grinta fosca, finché uno sparo in testa non vi stende. (Celati, Sonetti 14)

Altro tema ricorrente nei componimenti di Vecchiatto e particolarmente rilevante nel caso politico italiano degli anni Duemila è quello del potere mediatico. La televisione viene messa a nudo nella sua duplice funzione, indispensabile al potere, di distrazione generale e, allo stesso tempo, diffusione dei valori su cui 
si erge questa società dello spettacolo, gli stessi del capo che comanda la nazione $e^{28}$ (ottimismo forzato, ${ }^{29}$ invito al consumo ${ }^{30}$ e all'arricchimento furbesco):

\section{Sonetto sulla televisione italiana}

Signori, quelli che vi dan da bere il cicaleccio di spettacolistiche trasmissioni per passar le sere con frescate politiche o calcistiche, sono forse le più caratteristiche facce di criminali da vedere, più tristi assai delle bande teppistiche, più torvi di chi sta nelle galere. E voi vi sorbite con seral clistere il succo marcio delle lor casistiche, la morfina che vi fa stravedere per vacue sceneggiate scandalistiche. (Celati, Sonetti 33)

\section{Secondo sonetto sulla televisione italiana}

Bastano trenta serate davanti

all'apparecchio di televisione

e non avrete più alcuna passione

\footnotetext{
${ }^{28}$ Per una analisi della stretta connessione tra il sistema valoriale del Berlusconi politico e il suo impero mediatico si vedano Berlusconismo. Analisi di un sistema di poteree Berlusconi. Ambizioni patrimoniali in una democrazia mediatica di Paul Ginsborg,

${ }^{29}$ Cfr. il sonetto 10: "Voi contro i disperati ed i depressi / elevate barriere d'ottimismo, / [...] / Ma il vostro sperare, e vantaggi annessi, / per lo più sono puro fanatismo: / più soldi, più potere, più successi, / in gare d'ansia nel mar del solipsismo." (Celati, Sonetti: 72).

${ }^{30}$ Cfr. il sonetto 9: "Consuma, consuma e andrai in paradiso, [...] Continua a consumare e fai buon viso / a fregature, debiti e al demente / obbligo di star sempre sull'avviso, perché del nuovo non ti sfugga niente" (Celati, Sonetti 26) e il 33: "Hanno ficcato in testa a tutti quanti / che il nuovo sempre sia cosa migliore, / e in massa vedi ovunque gli zelanti / vestire i panni dell'adoratore / d'ogni gadget ch'è nuovo e un po' più avanti / [...] / Il Badalucco ne conquista tanti / che più non resta un onesto obbiettore: / s'inchinan tutti ai nuovi fabbricanti / cantando gli inni del consumatore" (Celati, Sonetti 73).
} 
per le cose sottili e dissonanti.

Ammirerete sol l'ostentazione,

le becere battute accattivanti,

il culo e le tette debordanti

di un'oca stridula da esposizione. ${ }^{31}$

E vi parranno davvero esaltanti

le grida pubblicitarie di un marpione,

che voi applaudite tutti in soggezione,

nello sfarzo di luci là davanti.

Luci d'una vita ove tutto s'indora,

nel borghese comfort della malora. (Celati, Sonetti 34)

La televisione produce un conformismo generalizzato, le cui conseguenze politiche sono rese esplicite in un altro sonetto, il 40:

40. Sul dibattito democratico in Italia

Sono rimasto tre sere davanti

all'apparecchio di televisione;

Badalucco ha parlato a profusione, non ho sentito voci dissonanti.

Ha parlato solo lui in mezzo a tanti;

lodando la libertà d'espressione,

e tra bordate di applausi scroscianti,

alla fine si è dato anche ragione.

"Godetevi la vita!," ecco il sermone

che attira i battimani dei votanti.

"Vacanze, libertà, competizione!

Siate liberi e ricchi tutti quanti!"

Chiotti chiotti, senza dire beo,

gli oppositori si accodano al corteo. (Celati, Sonetti 85)

${ }^{31}$ Cfr. Recita: "Tutti col faro dei loro più alti pensieri puntato soltanto sul culo e le tette d'una povera oca che mostra il culo e le tette in televisione! [....] dove una povera oca che mostra il deretano in televisione è considerata centomila volte più importante di Dante, Shakespeare, Leopardi, mi dica lei cosa c'è da sperare?” (Celati, Recita 54-55). 
Come numerosi altri interventi di Celati, non solo di questi anni, ${ }^{32}$ Sonetti coinvolge anche la società cosiddetta colta, chiusa nella sua autoreferenzialità e responsabile alla pari, secondo Celati-Vecchiatto, di questo sfascio morale e politico:

26. Risposta a un professore universitario che si compiaceva di vilipendere Vecchiatto senza averlo mai visto recitare

O grande ispiratore di tromboni trombon tu stesso, paturnia, clistere, maestro di quelli che credono avere la sapienza masticando citazioni. Protervo e astruso, la via da tenere tu additi alla maniera dei sermoni: l'unica - dici - per non andare a tentoni nella notte delle vacche tutte nere ${ }^{33}$ [...]

Pastoria greve di falsa certezza, ecco il delirio della saputezza. (Celati, Sonetti 57)

39. Sonetto dedicato ai critici

Io non ho mai capito se i critici che scrivono sui diversi giornali sono una sola persona con mitici nomi vari, famosi e nazionali [...]

Certo è che i loro articoli rachitici, smerciati solo per scopi venali,

\footnotetext{
32 Tra i tanti esempi possibili, si veda il racconto "I lettori di libri sono sempre più falsi," in Gianni Celati, Quattro novelle sulle apparenze (61-95).

${ }^{33}$ Cfr. la nota inserita da Celati (o da Vecchiatto? Dall'“autore" o dal "curatore"?) al termine del sonetto, dove si specifica che la frase "la notte delle vacche tutte nere," derivata dell'Hegel della Fenomenologia dello spirito, era stata "molto usata da intellettuali di sinistra, prima di accodarsi alla marcia trionfale del Badalucco" (Celati, Sonetti 57).
} 
e con pensieri - diciam pure - stitici,

sono i nostri oracoli nazionali. (Celati, Sonetti 84) 34 $^{34}$

L'ultimo componimento, il 51, è un elogio della povertà onesta contro le "ricchezze ladre" (Celati, Sonetti 106). Il congedo di Vecchiatto dai suoi sonetti mette in scena un exemplum vitae da "clandestino in latitanza" (Celati, Sonetti 72) in una società come quella descritta fino a qui. È la parabola di una vita che mostra a pieno la debolezza e la contraddizione, l'ambizione, il fallimento e la sconfitta, qui come in Recita, ma che è stata capace di tenersi il più possibile alla larga dalla società del consumo e dello spettacolo:

Da non so dove è venuto Vecchiatto, nato a Venezia per error del padre; l'attrazione del seno di sua madre fu il primo amore che lo rese matto. Mai allettato da ricchezze ladre, fu però dalla carne molto attratto; ciò lo rese geloso e anche distratto, finché Carlotta lo rese padre.

Viaggiatore, vagò e visse d'accatto

Senza imbarcarsi mai in losche squadre; adorò l'aria e odiò le teste quadre, uscì di scena in patria all'ultim'atto. Polvere che s'impasta quando piove, e Vecchiatto è tornato non so dove. (Celati, Sonetti 106)

Se in Recita lo sproloquiare di Vecchiatto contro tutti è continuamente stemperato dagli interventi, insieme affettuosi e ironici, della moglie Carlotta e dalle risate (e dalle dormite!) dei rarefatti e casuali spettatori che giungono in teatro, in

${ }^{34}$ Cfr. la trascrizione dell'incontro con Celati organizzato nel 2008 dal Circolo dei lettori di Torino dove, a una domanda sul senso della critica oggi, risponde: "Dunque, ci sono questi che scrivono sul giornale che se la prendono ora con uno ora con l'altro, anch'io ho preso un sacco di legnate, specie da Goffredo Fofi, ora, spero, si sarà sfogato. Io non capisco chi scrive contro qualcuno o contro un libro, posso solo intuire che abbia bisogno di sfogarsi per un qualche suo problema. [...] La critica io la concepisco come un lavoro di affezione, vale solo se presuppone un rapporto di affetto con l'autore. Ha un senso se è un proporre ad altre persone la propria amicizia con quell'autore" (Celati, "Incontro con Gianni Celati"). 
Sonetti l'invettiva si fa più netta ed elaborata, non solo nei componimenti poetici, ma anche nelle parti in prosa in cui di Vecchiatto racconta la propria vita. La critica della società messa in bocca da Celati a Vecchiatto va, dunque, presa sul serio ${ }^{35}$ $\mathrm{e}$, se è vero che ad essa si fondono altre tematiche, fin dal principio si ricorda che il ragionamento sull'“Italia trista” è il motivo centrale del libro:

Questi sonetti parlano del vivere e del morire, dell'amore e del disamore, della nostra cecità e della luce immaginativa, della vita terrestre e della vocazione teatrale che ci guida attraverso il buio della mente. Ma soprattutto parlano d'una "Italia trista," che non sa cosa sia vergogna, e dell'ottimismo obbligatorio più bigotto, della vita come target aziendale, dell'opulenza come insolenza, dell'italicismo come stupidità di comodo, e dell'ineluttabile ansia prodotta dal "borghese comfort della malora" (parole di Vecchiatto). ${ }^{36}$

Sul finale, anche i Sonetti sono ricondotti a uno sfogo, in una deminutio che va a giustificare il tono talvolta eccessivamente aspro ${ }^{37}$ di certi componimenti in una specie di funzione terapeutica ${ }^{38} \mathrm{e}$, a sua volta, sociale:

49.

Questi sonetti vado componendo quando il vuoto non riesco più a soffrire, dunque per sfogo mi metto a farcire con rime fatue il nervosismo orrendo.

Senza pensare a cosa devo dire,

\footnotetext{
${ }^{35}$ Celati andrà a lungo a leggere i sonetti in pubblico, in Italia e all'estero, anche distribuendoli gratuitamente come fascicoli sciolti. Cfr. i ringraziamenti alla fine del libro (Celati, Sonetti 107-108).

${ }^{36}$ Cfr. la Premessa del curatore a firma di Gianni Celati (Sonetti 8).

37 Si veda anche il sonetto 2 dove, dopo una serie di accuse furibonde, Vecchiatto scrive una coda con cambiamento di tono: "Vorrei parlarvi con più cortesia, / vorrei mostrarvi la nostra fratellanza, / perché siamo tutti nella stessa scia, / e non ne possiam più di questa danza” (Celati, Sonetti 33).

${ }^{38}$ Sulla funzione terapeutica della letteratura nell'idea di Celati cfr. Anna Maria Chierici, La scrittura terapeutica. Saggio su Gianni Celati.
} 
alla cieca mi lancio combattendo, contro mulini a vento, e anche prendendo spesso pose da grand'uom nelle mie ire. Ma questi sfoghi vani cui attendo Spero possano dentro di me guarire le piaghe d'un patetico languire; cosicché ben sfogato, ancor vivendo, ogni umor nero riesca in me a dissolvere, riconciliato col mondo di polvere. (Celati, Sonetti 104)

Celati affida il suo più diretto intervento politico, negli anni ruggenti del berlusconismo (1996-2010), alla voce dell'attore Attilio Vecchiatto, creando una biografia fantastica insieme a Enrico De Vivo e dando nuova linfa a un ruolo sociale che l'immaginazione letteraria può assumere. Non a nomi attuali o casi cronachistici si fa riferimento, ma al Badalucco, ${ }^{39}$ figura esemplare, "categoria dello spirito" che contiene in sé tutte le tendenze dell'italicismo e va oltre il governante di turno, ma riguarda tutti:

Al bar Raiola ho sentito una sera qualcuno chiedergli: "Scusi tanto, maestro, ma chi sarebbe questo Badalucco?.” Al che Attilio è salito su una sedia e ha fatto un gran discorso ai clienti del bar, dicendo che Badalucco è dentro di noi, non è un personaggio iscritto all'anagrafe, ma una categoria dello spirito. "Dunque quella domanda," diceva, "dovete farla a voi stessi: chi è Badalucco? Sono anch'io un Badalucco o un suo collaboratore?" (Celati, Sonetti 39)

Il concetto di Badalucco come "furbone che conosciamo tutti" (Celati, Sonetti 8) si lega nel pensiero di Celati a una definizione usata da Cesare Zavattini, secondo cui si doveva "defurbizzare l'Italia": "Diceva Cesare Zavattini: 'Non è facile defurbizzare un ambiente, perché la furbizia permette di ottenere il massimo col minimo prezzo. Però poi non è vero: la furbizia è quella cosa che crea tutti gli intralci

\footnotetext{
${ }^{39}$ A testimoniare una volta di più la straordinaria coerenza interna dell'opera di Celati, va notato che il termine ricorre anche nel primo dei libri di Celati, Comiche, pur nella sua riscrittura del 1972-73: "E laddove gli angariati protestino l'uomo statale deve rispondere seccamente: Badalucco, citrullo, cos'hai da dire?" (Celati, Comiche 166).
} 
e i pasticci, e un numero sterminato di telefonate inutili'” (Celati, Conversazioni 146). ${ }^{40}$

Accanto al menzionato recupero della dimensione sociale dell'immaginazione, si assiste, nel Celati di questi anni, a un ricorso alle forme letterarie tradizionali e all'esplicitazione di modelli e fonti provenienti dalla tradizione letteraria italiana, alla quale Celati si richiama continuamente e dichiaratamente. Oltre alla forma sonetto, all'ampio utilizzo di lessico dantesco, alle citazioni shakespeariane che scandiscono e danno forma alla Recita, il riferimento principale per Recita e Sonetti appare, ancora una volta, Leopardi. ${ }^{41}$ Il testo faticosamente messo in scena da Vecchiatto nel primo libro è definito dall'attore "un'operetta morale" e il nome del recanatese ricorre in un'altra scena, contrapposto allo spettacolo televisivo di corpi femminili nudi (Celati, Recita 55). Ma è in Sonetti che Leopardi compare in maniera più diretta ed esplicita. Gianni Celati diventa personaggio nella fantastica "Testimonianza autentica sull'attore Vecchiatto" raccontata da Enrico De Vivo: l'ultima volta che quest'ultimo avrebbe visto Vecchiatto, che per mesi aveva soggiornato presso di lui ad Angri (Salerno), insieme alla moglie Carlotta, sarebbe stata a Torre del Greco, alle pendici del Vesuvio, nel 1992. Celati e Vecchiatto si incontrano, in un luogo più che mai leopardiano e legato all'ultimo dei componimenti poetici del recanatese: "Proposi di visitare la Villa delle Ginestre, ultima residenza di Giacomo Leopardi, una villa stile impero, isolata tra i prati di fronte all'erta del Vesuvio, dove il nostro poeta, affetto da varie malattie, miserando d'aspetto e d'umore, fu ospitato da Antonio e Paolina Ranieri fino al giorno della morte" (Celati, Sonetti 97).

Nel racconto di De Vivo, prima Celati canta davanti a Vecchiatto una sua operina per musica dedicata alla morte di Leopardi, poi è il grande attore a recitare versi tratti dai canti "Alla sua donna" e "I nuovi credenti." La Villa delle Ginestre era chiusa per la pausa pranzo e i tre non erano potuti entrare. È qui che De Vivo e Celati vedono Attilio Vecchiatto un'ultima volta, mentre si dirige verso il mare, camminando e poi correndo. Vecchiatto, dunque, sparisce nei luoghi della Ginestra leopardiana, al termine di quello che ha avuto tutta l'aria di un corteo

\footnotetext{
40 Zavattini è una figura centrale nel pensiero di Celati sul cinema (Cfr. "Documentari imprevedibili come i sogni”).

${ }^{41}$ Leopardi viene recitato nel primo film di Celati, Strada provinciale delle anime (1991) e ad esso fa riferimento John Berger (peraltro citato in Sonetti 51) in Case sparse (2003), di cui è la principale voce narrante.
} 
funebre e di un accompagnamento sereno al "nulla che sempre lo attese" (Celati, Sonetti 66), così come nella lezione di Leopardi e, anche, in quella di Celati.

Al momento dell'uscita dei Sonetti, le premesse etiche del riferimento a Leopardi erano state rese esplicite, oltre che nel manifesto dei redattori di Zibaldoni citato in apertura di questo studio, in un articolo a firma di Celati e apparso anch'esso sull'Unità nel marzo del 2004. ${ }^{42}$ Qui Celati spiega la necessità del pensiero di Leopardi rispetto all'attualità ("Leopardi è un pensatore che in questo momento è essenziale per andare avanti di giorno in giorno" Celati, "Il desiderio" 1) dominata da quell'ottimismo totalitario che sarà, lo abbiamo visto, uno dei bersagli principali dei Sonettit. ${ }^{43}$ Il pensiero leopardiano e la letteratura che ad esso si richiama, non necessariamente per via diretta, sono intrinsecamente politici: "la letteratura ci riafferma questo niente che siamo. E solo perché siamo un niente noi abbiamo bisogno di stare assieme. Non c'è idea di comunità possibile se non a partire dal fatto che siamo un niente, ciascuno di noi è un niente." (Celati, "Il desiderio" 1)

Celati cita lo Zibaldone leopardiano in quello che considera il "punto di partenza più rivoluzionario" della sua filosofia, una difesa dell'importanza delle illusioni, rispetto ai continui "richiami alla 'concretezza' da parte dei politici" (che "fanno veramente ridere"), e una dichiarazione del niente che siamo:

Dice Leopardi: "Il più solido piacere di questa vita è il piacer vano delle illusioni... Io considero le illusioni come una cosa in certo modo reale stante ch'elle sono ingredienti essenziali del sistema della natura umana, e date dalla natura a tutti quanti gli uomini, in maniera che non è lecito spregiarle come sogni di un solo, ma propri veramente dell'uomo e voluti dalla natura, e senza cui la vita nostra sarebbe la più misera e barbara cosa ecc. Onde sono necessari ed entrano sostanzialmente nel composto ed ordine delle cose." (Zibaldone 51)

Ne viene fuori un'idea di letteratura - e della politicità che essa porta con sé - profondamente diversa da quella di altri testi e autori che si considerano o

\footnotetext{
${ }^{42}$ Il quotidiano l'Unità, diretto da Furio Colombo prima (2001-2005), da Antonio Padellaro poi (2005-2008), e con Marco Travaglio tra le sue firme, era al tempo il giornale più dichiaratamente anti-berlusconiano.

${ }^{43}$ Si noti anche qui il chiaro riferimento a Berlusconi, sempre senza nominarlo: "quell'ottimismo che quel tale lì per mezz'ora stilò come programma del suo partito" ("Il desiderio infinito").
} 
vengono considerati impegnati. È un impegno che non prevede nessun "ritorno al realismo": "La gente al giorno d'oggi crede che la letteratura, parlare o fare letteratura sia fare pubblicità a qualcosa. La letteratura è muta, non fa pubblicità a niente, non serve a niente, la letteratura ci riafferma questo niente che siamo." (Celati, "Il desiderio" 1). Nel settembre del 2009, Zibaldoni pubblica uno scritto di Celati che fa da prefazione al nuovo libro di Enrico De Vivo, Divagazioni stanziali. ${ }^{44} \mathrm{Il}$ testo contrappone il modo di scrivere di De Vivo a quello dei libri al momento più in voga ("il suo libro è molto diverso dai libri che si scrivono e pubblicano con grande sfarzo di elogi pubblicitari” Celati, "Devo dirglielo") e il riferimento polemico a Roberto Saviano ${ }^{45}$ è evidente: "Devo dirglielo che va bene così, anche se non scriverà mai Sodoma 1 e Gomorra 2. Ma ci sono gli altri che lo faranno, quelli con tutte le giustificazioni e le carte in regola" (Celati, "Devo dirglielo").

Sono ragionamenti che Celati ribadisce più volte e che richiamano, prima di tutto, un testo capitale per chi ne studia l'opera, ovvero il saggio "Le posizioni narrative rispetto all'altro," nato da una conferenza tenuta all'Università di Caen nel 1994 e poi pubblicato su Nuova Corrente nel 1996, lo stesso anno di Recita. Celati contrappone qui le tendenze scrittorie naturalistiche e realistiche, da Zola alla "letteratura ideologica" di Jean Paul Sartre, Iris Murdoch e Alberto Moravia (Celati, "Le posizioni" 10), fino al "romanzo industriale contemporaneo," (Celati, "Le posizioni" 10) a quelle narrazioni in cui il rapporto con il lettore o ascoltatore è costruito non su descrizioni impositive del fatto concreto, ma su ampi cerimoniali che fanno da preludio a racconti che seguono la naturale disposizione alla divagazione fantastica, come nella tradizione della novella o del poema cavalleresco. Alla supposta oggettività, all'ostentazione del fatto concreto a scopo informativo o di svolgimento di una tesi, Celati predilige un "narrare naturale" fatto di libere divagazioni immaginative la cui intima eticità riposa nella posizione assunta rispetto al lettore, nella "connivenza fantastica, di simpatia immaginativa con l'ascoltatore" (Celati, "Le posizioni" 11) e nel continuo rapportarsi con un altro-da-sé, negato invece, secondo Celati, dalla rigidezza linguistica e di pensiero

\footnotetext{
${ }^{44}$ Del libro di De Vivo, Celati elogia la modestia, l'abbassamento di tono, la lontananza da "quelli che non vogliono sentirsi sbagliati" ("Devo dirglielo"), l'amore per il niente di speciale (cfr. "Lode del niente di speciale," in Celati, Sonetti 47), per le divagazioni e per il carattere gratuito del suo scrivere storie. Cfr. Gianni Celati, “Devo dirglielo a Enrico De Vivo”).

${ }^{45}$ Cfr. Roberto Saviano, Gomorra: Viaggio nell'impero economico e nel sogno di dominio della camorra.
} 
dello "scrittore professionista," (Celati, "Le posizioni" 11 ) 46 $^{46}$ chensa al lettore non come individuo specifico ma come dato statistico, un lettore disinformato in attesa di ricevere il messaggio dell'autore. Al contrario, l'immaginazione permette di stabilire un legame affettivo con l'altro, perché fa leva su una capacità narrativa che non è sapere specialistico, ma predisposizione umana generale, che ognuno apprende nel momento in cui si impara la lingua materna ("tutti i parlanti d'una lingua si intendono immaginativamente senza bisogno di tante spiegazioni" Celati, "Le posizioni" 11). È il "benedetto senso comune" (Celati, "Le posizioni" 18) che "ci lega agli altri nei pensieri a distanza, anche nel quadro di una separazione generale degli individui come quella in cui viviamo" (Celati, "Dialogo sulla fantasia con Massimo Rizzante"). ${ }^{47}$

L'immaginazione, dunque, non prevede nessuna fuga o rifugio dal reale, ma si configura in Celati, qui sulla scorta del pensiero di Giambattista Vico e del De Anima di Aristotele, come supremo metodo conoscitivo e interpretativo, a cui ricorriamo continuamente:

Il fatto è che noi ci serviamo della fantasia tutti i momenti per interpretare le cose, cercando di capire quello che è fuori dalla nostra portata; e tutto il nostro sistema emotivo dipende da come immaginiamo ciò che non è sotto i nostri occhi. Quando abbiamo paura, quando siamo a disagio, quando siamo gelosi, quando facciamo progetti, entra in gioco l'atto di fantasticare [...]. Il fantasticare è così assiduo che lo diamo per scontato. Però se si inceppa abbiamo un campanello d'allarme, che è la noia. (Celati, "Dialogo sulla fantasia con Massimo Rizzante")

Fantasia non è, quindi, sinonimo di irrealtà, ma "vasta memoria collettiva" e straordinaria forza conoscitiva, di cui certi scrittori contemporanei sembrano essersi voluti sbarazzare, come "ospedalizzati in questo mondo e nella cosiddetta 'realtà,' costretti ad attingere dall'attualità più stringente e parlare di "qualcosa di

\footnotetext{
${ }^{46}$ Alla figura dello scrittore professionista, Celati contrappone dunque i "vecchi raccontatori di favole, i vecchi rapsodi, i cantastorie, e anche quei vecchi personaggi che raccontavano la loro vita per il gusto di narrare, spesso in modo straordinario" (Celati, "Le posizioni” 11).

${ }^{47}$ Un'altra versione dell'articolo, ampliata, è disponibile su Zibaldoni (“Dialogo sulla fantasia”).
} 
drammatico su questo mondo, sulle 'realtà,' per poter essere presi seriamente" (in O’Brien 12). ${ }^{48}$

L'opera di Celati degli anni Duemila ${ }^{49}$ mostra una chiara matrice etico-politica, di reazione critica a fenomeni quali l'invito continuo al consumo, la dittatura del nuovo, la vita democratica come marketing elettorale, l'ottimismo obbligatorio, la rimozione totalitaria del perdente, del vecchio e del cadente, l'esposizione dei corpi come merce, la retorica del successo che passa attraverso l'arricchimento furbesco, ecc. Il ciclo di Vecchiatto, composto da Recita e Sonetti, mentre recupera le forme della tradizione letteraria italiana, pur all'interno di un impianto decisamente postmoderno, ospita un'ampia invettiva nei confronti dell'Italia degli anni Novanta e Duemila, dove l'impegno emerge in maniera diretta. Contro gli aspetti più retrivi della vita pubblica italiana, Celati propone una sorta di fratellanza affettiva che vede la fragilità umana come base politica della necessità di costituirsi in comunità. Di questo, la sua idea stessa di letteratura ci appare portatrice: non una scrittura appiattita sulla cronaca giornalistica, ma una narrazione immaginativa che si appella alla fantasia come risorsa interpretativa che appartiene a tutti, non solo - e non tanto - agli scrittori di professione, e che è continuamente rivolta alla cura e all'ascolto dell'altro-da-sé.

Gli scritti teorici e la collaborazione alla rivista online Zibaldoni permettono di situare tale critica della società contemporanea in un quadro più ampio, che mostra come l'opera di Celati, fin dagli esordi animata da fondamenti etici, abbia rappresentato un modello di impegno alternativo a quello del "ritorno al reale." I numerosi richiami all'opera leopardiana, sia negli scritti di Celati che nell'attività e nei manifesti teorici di Zibaldoni, ci hanno fatto parlare di una vera e propria "linea leopardiana dell'impegno," definizione che, riprendendo il titolo di un articolo di Celati sulla prosa dello Zibaldone di Leopardi (Celati, "La linea"), ci sembra adatto a indicare le forme di impegno frammentario, post-ideologico, eppure estremamente radicale di cui Celati si fa promotore e protagonista e che ci

\footnotetext{
48 "La narrativa d'oggi è ormai un'appendice dell'informazione giornalistica. È difficile trovare un romanzo d'oggi che non si appelli all'attualità" ("Dialogo sulla fantasia").

${ }^{49}$ Il discorso sviluppato fin qui può estendersi a tutta l'opera di Celati degli anni Duemila, dai racconti di Cinema naturale a Fata Morgana fino al Celati regista (Case sparse e Diol Kadd) e al ciclo dei Costumi degli italiani (comprensivo di Vite di pascolanti e Selve d'amore) dove il richiamo a Leopardi è particolarmente evidente fin dal titolo. Di questi stessi anni è anche un altro testo a forte connotazione politica: Gianni Celati, Ma come dicono di vivere cosi.
} 
paiono particolarmente incisive, proprio per il ricorso a quel patrimonio collettivo rappresentato dall'immaginazione archetipica e narrativa.

Michele Ronchi Stefanati, University College Cork

\section{Opere Citate}

Alfabeta 21.1 (2010).

Antonello, Pierpaolo. Dimenticare Pasolini. Intellettuali e impegno nell'Italia contemporanea. Milano-Udine: Mimesis, 2012.

Antonello, Pierpaolo, e Florian Mussgnug (a cura di). Postmodern Impegno: Ethics and Commitment in Contemporary Italian Culture. Oxford: Peter Lang Pub Inc, 2009.

Barenghi, Mario. "Congetture su un dissenso." Riga 14. Alì Babà. Progetto di una rivista 1968-1972. A cura di Mario Barenghi e Marco Belpoliti. Milano: Marcos y Marcos, 1998. 13-23.

Barenghi, Mario, e Marco Belpoliti. (a cura di). Riga 14. Alì Babà. Progetto di una rivista 1968-1972. Milano: Marcos y Marcos, 1998.

Bartezzaghi, Stefano. Scrittori giocatori. Torino: Einaudi, 2010.

Baudrillard, Jean. Il delitto perfetto. La televisione ha ucciso la realtà? Milano: Raffaello Cortina Editore, 1996.

Benedetti, Carla. Il tradimento dei critici. Torino: Bollati Boringhieri, 2002.

Berardinelli, Alfonso. Casi critici. Dal postmoderno alla mutazione. Macerata: Quodlibet, 2007.

Belpoliti, Marco, e Marco Sironi (a cura di). Riga 28. Gianni Celati. Milano: Marcos y Marcos: 2008.

Binetti, Vincenzo. "Marginalità e appartenenza: la funzione dell'intellettuale tra sfera pubblica e privato nell'Italia del dopoguerra." Italica 74.3 (1997): 361-374.

Bolongaro, Eugenio, Mark Epstein, e Rita Gagliano (a cura di). Creative Interventions: The Role of Intellectuals in Contemporary Italy. Newcastle: Cambridge Scholars Publishing, 2009.

Bonsaver, Guido, Martin McLaughlin, e Franca Pellegrini (a cura di). Sinergie Narrative. Cinema e letteratura nell'Italia contemporanea. Firenze: Franco Cesati, 2008. 
Botta, Anna. "The Alì Babà project (1968-1972): Monumental History and the Silent Resistance of the Ordinary." Italianistica Ultraiectina 1 (2006). <https://dspace.library.uu.nl/handle/1874/29264> Consultato il 3 dicembre 2014.

Burns, Jennifer. Fragments of impegno. Interpretations of Commitment in Contemporary Italian Narrative 1980-2000. Leeds: Northern UP, 2001.

Calvino, Italo. "Lo sguardo dell'archeologo." Saggi 1945-1985. A cura di Mario Barenghi. Milano: Mondadori, 1995. 324-327.

Celati, Gianni. "Bollettino del diluvio universale — Pantomima in due atti." "Diluvi", numero tematico di Elephant \& Castle. Laboratorio dell'immaginario 1 (aprile 2010). <http://cav.unibg.it/elephant_castle/web/saggi/em-bollettino-del-diluvio-universale-em-pantomima-in-due-atti/19> Consultato il 2 dicembre 2014.

. Comiche. Macerata: Quodlibet, 2012.

. Conversazioni del vento volatore. Macerata: Quodlibet, 2011.

. "Devo dirglielo a Enrico De Vivo." Zibaldoni e altre meraviglie 15 settembre 2009. <http://www.zibaldoni.it/2009/09/15/devivo-2/> Consultato il 29 maggio 2014.

. "Dialogo sulla fantasia." Zibaldoni e altre meraviglie 19 settembre 2005. <http://www.zibaldoni.it/2005/09/19/dialogo-sulla-fantasia/> Consultato il 6 dicembre 2014.

. "Dialogo sulla fantasia con Massimo Rizzante." Griseldaonline 7 (20072008). <http://www.griseldaonline.it/temi/a-rovescio/celati-dialogo-sullafantasia-con-massimo-rizzante.html> Consultato il 13 maggio 2014.

. "Documentari imprevedibili come sogni. Intervista di Sarah Hill a Gianni Celati." Documentari imprevedibili come i sogni. Il cinema di Gianni Celati. A cura di Nunzia Palmieri. Roma: Fandango, 2011. 71-85.

. Finzioni occidentali. Fabulazione, comicità e scrittura. Torino: Einaudi, 1975.

. "Il desiderio infinito." Zibaldoni e altre meraviglie 28 marzo 2004. <http://www.zibaldoni.it/2004/03/28/il-desiderio-infinito/> Consultato l'11 giugno 2014.

. "Il progetto Alì Babà, trent'anni dopo." Riga 14. Progetto di una rivista 1968-1972. A cura di Mario Barenghi e Marco Belpoliti. Milano: Marcos y Marcos, 1998. 313-321. 
. "Incontro con Gianni Celati (primo tempo)." La poesia e lo spirito. Potrà questa bellezza rovesciare il mondo? 3 marzo 2008. <http://lapoesiaelospirito. wordpress.com/2008/03/03/incontro-con-gianni-celati-primo-tempo/>

. "La linea leopardiana della prosa." Zibaldoni e altre meraviglie 28 febbraio 2003. <http://www.zibaldoni.it/2003/02/28/la-linea-leopardiana-della-prosa/> Consultato il 2 dicembre 2014.

. "Le posizioni narrative rispetto all'altro." Nuova corrente 43 (1996): $2-18$.

- "Letteratura come accumulo di roba sparsa. Conversazione con Marco Belpoliti e Andrea Cortellessa." Riga 28. Gianni Celati. A cura di Marco Belpoliti e Marco Sironi. Milano: Marcos y Marcos, 2008. 25-37.

. L'Orlando innamorato raccontato in prosa. Torino: Einaudi, 1994.

. Ma come dicono di vivere cosi. Viadana: FUOCOfuochino, 2009.

- Narratori delle riserve. Milano: Feltrinelli, 1992.

- Parlamenti buffi. Milano: Feltrinelli, 1989.

- Quattro novelle sulle apparenze. Milano: Feltrinelli, 1987.

. Recita dell'attore Vecchiatto nel teatro di Rio Saliceto, Milano: Feltrinelli, 1996.

. Sonetti del Badalucco nell'Italia odierna. Milano: Feltrinelli, 2010.

Cesari, Severino, 'L'artigiano delle parole', il Manifesto, 8 marzo 1989, p. 15.

Chierici, Anna Maria. La scrittura terapeutica. Saggio su Gianni Celati. Bologna: Archetipo Libri, 2011.

De Vivo, Enrico. Divagazioni stanziali. Verona: QuiEdit, 2009.

De Vivo, Enrico, e Gianluca Virgilio. "La letteratura condivisa: il piacere di fare politica." Zibaldoni e altre meraviglie 31 gennaio 2004. <http://www.zibaldoni. it/2004/01/31/la-letteratura-condivisa-il-piacere-di-fare-politica/> Consultato il 12 maggio 2014.

Di Stefano, Paolo. "La scure di Fofi nel giardino di Cavazzoni e Celati." Corriere della Sera 14 febbraio 1996. <http://archiviostorico.corriere.it/1996/febbraio/14/scure_Fofi_nel_giardino_Cavazzoni_co_0_9602145545.shtml.> Consultato il 1 dicembre 2014.

Donnarumma, Raffaele. "Ipermodernità: Ipotesi di congedo dal postmoderno." Allegoria 64 (2011): 15-50.

. “Postmoderno italiano: Qualche ipotesi." Allegoria 43 (2003): 56-85.

Francioso, Monica. "Impegno and Alì Babà: Celati, Calvino and the Debate on Literature in the 1970s." Italian Studies 64 (2009): 105-119. 
Gaber, Giorgio. Quando parla Gaber. Pensieri e provocazioni per l'Italia di oggi. A cura di Guido Harari. Milano: Chiare lettere, 2011.

Ginsborg, Paul. Berlusconi. Ambizioni patrimoniali in una democrazia mediatica. Torino: Einaudi, 2003.

- Berlusconismo. Analisi di un sistema di potere. A cura di e con Enrica Asquer. Roma-Bari: Laterza, 2011.

Iacoli, Giulio. La dignità di un mondo buffo. Intorno all'opera di Gianni Celati. Macerata: Quodlibet, 2011.

. "Lo sguardo in macchina. Note sull'immaginario cinematografico di Gianni Celati." Sinergie narrative. Cinema e letteratura nell'Italia contemporanea. A cura di Guido Bonsaver, Martin MacLaughlin e Franca Pellegrini. Firenze: Franco Cesati Editore, 2008. 139-152.

Jansen, Monica, e Claudia Nocentini. "Alì Babà and Beyond: Celati and Calvino in the Search for 'Something More'." Italianistica Ultraiectina 1 (2006). <https://www.narcis.nl/publication/RecordID/oai\%3Adspace.library. uu.nl\%3A1874\%2F29219/uquery/Al\%C3\%AC\%20Bab\%C3\%A0\%20 and $\% 20$ Beyond $\% 3$ A $\% 20$ Celati\%20and\%20Calvino\%20in\%20the\%20 Search\%20for\%20\%E2\%80\%98\%E2\%80\%9CSomething\%20More\%E2\%80\%99/id/1/Language/NL> Consultato il 3 dicembre 2014.

"L'intellettuale e l'impegno." Numero di MicroMega 6 (2013).

Luperini, Romano. L'allegoria del moderno. Saggi sull'allegorismo come forma artistica del moderno e come metodo di conoscenza. Roma: Editori riuniti, 1990. . La fine del postmoderno. Napoli: Guida, 2005.

O’Brien, Flann. La miseria in bocca. Trad. Daniele Benati. Milano: Feltrinelli, 1987.

Re, Lucia. "Pasolini vs. Calvino One More Time: The Debate on the Role of Intellectuals and Postmodernism in Italy Today." Modern Language Notes 129.1 (2014): 99-117.

Rizzante, Massimo. Il geografo e il viaggiatore: variazioni su Italo Calvino e Gianni Celati. Pesaro: Metauro, 1993.

Rorato, Laura, e Marina Spunta (a cura di). Letteratura come Fantasticazione: In Conversazione con Gianni Celati. Lewiston, NY: Edwin Mellen Press, 2009.

Saviano, Roberto. Gomorra: Viaggio nell'impero economico e nel sogno di dominio della camorra. Milano: Mondadori, 2006.

Schwarz Lausten, Pia. "Impegno e immaginazione nell'opera di Gianni Celati." Letteratura come Fantasticazione: In Conversazione con Gianni Celati. A 
cura di Laura Rorato e Marina Spunta. Lewiston, NY: Edwin Mellen Press, 2009. 161-184.

Sironi, Marco. Geografie del narrare. Insistenze sui luoghi di Gianni Celati e Luigi Ghirri. Diabasis: Reggio Emilia, 2004.

Spunta, Marina. "Verso la foce con Gianni Celati: tra 'antropologia di popolazioni invisibili' e 'estetica del reincanto'." Sinergie narrative. Cinema e letteratura nell'Italia contemporanea. A cura di Guido Bonsaver, Martin MacLaughlin e Franca Pellegrini. Firenze: Franco Cesati Editore, 2008. 153-167.

West, Rebecca. Gianni Celati. The Craft of Everyday Storytelling. Toronto: U of Toronto P, 2000.

Wren-Owens, Elizabeth. Postmodern Ethics: The Re-Appropriation of Committed Writing in the Works of Antonio Tabucchi and Leonardo Sciascia 1975-2005. Newcastle: Cambridge Scholars Publishing, 2007. 